\title{
Persistence of a briefly presented visual stimulus in sensory memory
}

\author{
JESSE E. PURDY and DAVID G. EIMANN \\ Southwestern University, Georgetown, Texas 78626 \\ and \\ HENRY A. CROSS, JR. \\ Colorado State University, Fort Collins, Colorado 80521
}

\begin{abstract}
College students were given 12 trials in which two 4 by 4 matrices of letters were presented sequentially, each for $100 \mathrm{msec}$. On one-half of the trials, the second matrix was the same as the first, and on one-half of the trials, the second matrix differed from the first. Both the interval between the two presentations and the degree of difference between the second matrix and the first were manipulated systematically. Results indicated that when the difference between the second matrix and the first was either large or nonexistent, recognition was good. When the difference between the matrices was small, performance decreased significantly. The interstimulus interval variable was not significant. Results were discussed in terms of Sperling's (1960) findings and those of Cermak (1971) and Phillips (1974).
\end{abstract}

Sensory memory is thought to be the first stage in the information processing system and has three major characteristics. First, sensory memory has virtually unlimited capacity. That is, all sensory information impinging upon a certain sensory system is stored. Second, the information is held in sensory memory for a very brief period of time, usually less than $1 \mathrm{sec}$. Third, information in sensory memory is processed in terms of physical characteristics and is not categorized in terms of meaning until later. Evidence for these three characteristics comes from a series of experiments by Sperling (1960). In Sperling's paradigm, subjects were presented a matrix of letters for $50 \mathrm{msec}$ and asked to recall the entire matrix, the whole-report procedure, or an identified portion of the matrix, the partial-report procedure. Under the latter procedure, recall was significantly better and indicated that most of the incoming sensory information is available for recall, for a brief period of time. In Experiment 6, Sperling presented his subjects a matrix consisting of letters and numbers. When asked to report just the letters, or the numbers, performance was not different from that in the wholereport procedure, indicating that information in sensory memory is stored precategorically.

Although performance under the partial-report procedure is quite good, it is still not perfect. In fact, with a 3 by 4 matrix of letters, subjects could report only $76 \%$ correct. It is possible that the lower percentage is attributable to the partial-report procedure itself. Before the subject can report the letter, the process of pattern recognition must take place, since

Requests for reprints should be sent to the first author at the Department of Psychology, Southwestern University, Georgetown, Texas 78626 . information in sensory memory is precategorical. The invoking of another memory system complicates the task and may account for this finding. The present investigation was an attempt to remedy this situation by making the task one of recognition. Subjects were presented two slides, each for $100 \mathrm{msec}$, and were asked to press a response key that indicated whether the second slide was identical to or different from the first. With this method, since it is not necessary to invoke the process of pattern recognition, it may be possible to accurately assess the capacity of sensory memory proper. In this experiment, both the interstimulus interval (ISI) and the degree of difference between the two slides were systematically varied. It was hypothesized that as the ISI increased, performance would decrease. In addition, it was thought that the degree-of-difference variable would have no effect, since sensory memory is thought to be a high-capacity system and one in which even small differences should be detectable.

\section{METHOD}

\section{Subjects}

Fifty-eight male and female students enrolled in the introductory psychology course served. Students participated in order to meet course requirements.
Apparatus
The apparatus consisted of two Kodak Carousel projectors, each with a Lafayette (Model 43016) electronic tachistoscope attachment. In addition, a Lafayette repeat cycle timer (Model 51013) and two response keys (Model 58026) connected to a data recorder (Model 58004) were used. Subjects were presented a 4 by 4 matrix of white letters on a dark background. Two slides were presented sequentially in one of four ways: (1) The second slide was identical to the first, or (2) the second slide differed from 
the first by 1 letter, either 1 of the 4 inside letters or 1 of the 12 outside letters, or (3) the second slide differed from the first by 4 letters, either the 4 inside letters or one entire outside row or column, or (4) the second slide differed from the first by 16 letters, with the positions filled first by the unused letters, then by the other letters, randomly assigned to different positions. During blank periods, the screen and room were dark.

\section{Procedure}

Subjects were randomly assigned to one of four groups; the groups differed only with respect to the ISIs, which were $100,250,500$, or $5,000 \mathrm{msec}$. All subjects received six trials in which the second slide was the same as the first (same), two trials in which the second slide differed from the first by 1 letter (D1), two trials in which the second slide differed from the first by 4 letters (D4), and two trials in which the second slide differed from the first by 16 letters (D16). The duration of each slide was $100 \mathrm{msec}$. In addition, subjects were given two practice trials. The 12 nonpractice trials were randomly presented, and subjects were instructed that $50 \%$ of the trials would be "same" trials and 50\% would be "different" trials. The subject's task was to press a key marked "SAME" if it was thought the second slide was the same as the first and to press a key marked "DIFFERENT" if it was thought the second slide differed from the first. The experimenter called out "ready" prior to each trial.

\section{RESULTS}

The percentages of correct responses for all subjects are presented in Figure 1 as a function of ISI and the degree of difference between the second slide and the first. A split-plot analysis of variance on these data revealed no effect attributable to $\operatorname{ISI}[\mathrm{F}(3,54)=$ $2.17, \mathrm{p}>.05]$, a strong degree-of-difference effect $[F(3,162)=44.14, p<.01]$, and a significant interaction $[F(9,162)=2.29, p<.05]$. The degree-of-difference variable accounted for $35 \%$ of the variability, whereas the interaction accounted for only $5 \%$. Since the same analysis on arcsin-transformed scores required an identical interpretation, only nontransformed data are reported.

The mean percentages of correct responses for the ISIs of $100,250,500$, and $5,000 \mathrm{msec}$ were $64 \%, 71 \%$, $67 \%$, and $75 \%$, respectively. As mentioned, these means did not differ significantly. The mean percentages of correct responses for the four degrees of difference (same, D1, D4, and D16) were $80 \%, 43 \%, 60 \%$, and $96 \%$, respectively. A Newman-Keuls multiple-comparison test showed each of these means to be significantly different from each of the others. When all letters were different, performance was higher; when the first and second slides were the same, performance was also higher.

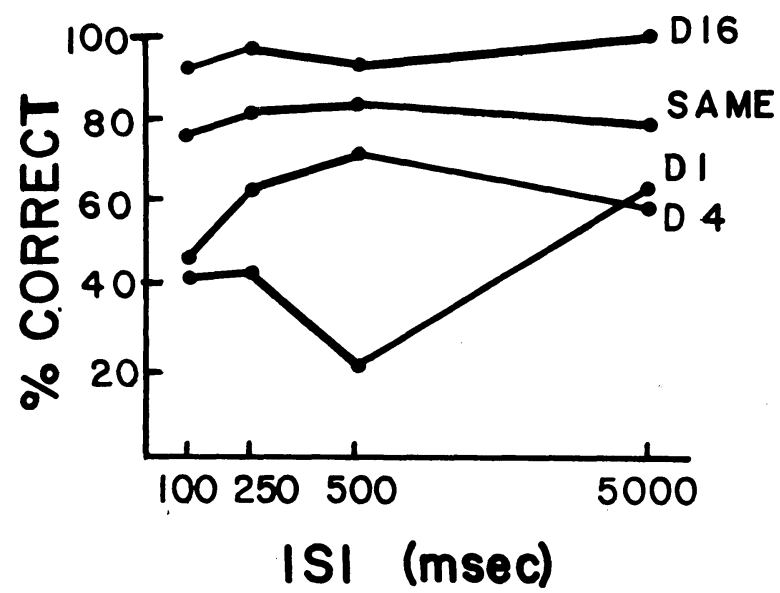

Figure 1. Percentages of correct responses as a function of ISI and degree of difference.

The D4 and D1 differences were the most difficult to detect and resulted in greatly reduced performance. Although the interaction was significant, a simple main effects test revealed that the differences were attributable largely to the degree-of-difference variable. The only significant difference among the ISIs occurred between $500 \mathrm{msec}$ and $5,000 \mathrm{msec}$ at D1.

Performance on D16 trials was excellent and may partially be explained by subjects' shifting to a more liberal criterion. Table 1 shows the hit rate (HR), false alarm rate (FAR), $\mathrm{d}^{\prime}$, and $\beta$ as a function of ISI and degree of difference. FARs were determined by using two randomly chosen "same" trials for D1, two for D4, and two for D16. Table 1 shows the D16 subjects to be more liberal and more sensitive than the D1 and D4 subjects.

\section{DISCUSSION}

Performance on the D16 trials was nearly perfect and provides partial support for the hypothesis that a recognition task would show that all incoming sensory information is processed and is retrievable. The poorer performance in the D1 and D4 conditions poses a problem for this hypothesis. It should not matter whether part or all of the stimulus is changed, if $100 \%$ of the information is processed. Bartram (1978) and Wilton and File (1975) provide a possible explanation for this finding. These researchers demonstrated that subjects group information that is held in posticonic visual storage. Bartram presented tachistoscopically 10 disks randomly placed in a

Table 1

Hit Rate (HR), False Alarm Rate (FAR), d', and $\beta$ as a Function of ISI (in Milliseconds) and Degree of Difference

\begin{tabular}{|c|c|c|c|c|c|c|c|c|c|c|c|c|}
\hline \multirow[b]{3}{*}{ ISI } & \multicolumn{12}{|c|}{ Degree of Difference } \\
\hline & \multicolumn{4}{|c|}{ D1 } & \multicolumn{4}{|c|}{ D4 } & \multicolumn{4}{|c|}{ D16 } \\
\hline & HR & FAR & $\mathrm{d}^{\prime}$ & $\beta$ & HR & FAR & $\mathrm{d}^{\prime}$ & $\beta$ & HR & FAR & $d^{\prime}$ & $\beta$ \\
\hline 100 & 42 & .15 & .83 & 1.70 & .46 & .35 & .29 & 1.08 & .92 & .23 & 2.15 & .50 \\
\hline 250 & .43 & .13 & .95 & 1.86 & .63 & .30 & .85 & .92 & .97 & .13 & 3.01 & .33 \\
\hline 500 & .21 & .14 & .27 & 1.32 & .71 & .21 & 1.36 & 1.17 & .93 & .14 & 2.56 & .59 \\
\hline 5000 & .63 & .22 & 1.10 & .79 & .59 & .16 & 1.23 & 1.63 & 1.00 & .25 & 4.38 & .01 \\
\hline
\end{tabular}


5 by 4 matrix and showed that the subjects generally recalled one or two chunks of spatially adjacent disks, consisting of three or four disks. It may be that such grouping also takes place in the sensory store. If the subject has only one or two groups of information in the sensory store, the probability of detecting a difference, or lack of difference, in the D16 and SAME conditions is better than in Conditions D1 and D4. This would account for the poorer performance in the D1 and D4 conditions and the better performance in the D16 and SAME conditions.

Perhaps the most surprising result concerns the negligible influence of the ISI variable. Performance for all four degrees of difference at $100 \mathrm{msec}$ was not significantly different from performance at $5,000 \mathrm{msec}$. These results are not consistent with those of Sperling (1960), who found that recall declined sharply after $1,000 \mathrm{msec}$. The explanation for this discrepancy may lie in the procedure itself. The use of the same-different recognition task is not new. Cermak (1971) and Phillips (1974) both have used a similar procedure. Cermak presented subjects not easily described shapes and showed a $78 \%$ recognition rate with an ISI of $1.5 \mathrm{sec}$ and $66 \%$ recognition rate with an ISI of $20 \mathrm{sec}$. The overall percentage of correct responses was $70 \%$. Phillips, using a 4 by 4 matrix of light and dark squares, showed better than $75 \%$ recognition with an ISI of $9 \mathrm{sec}$. Although these researchers were not using letters, the percentages of correct responses for long ISIs compare favorably with the present findings. It is interesting that evidence for an extended sensory memory only occurs when using the same-different recognition task. It was not shown in Sperling's recall paradigm. The higher performance at long ISIs found in the recognition forms of this task may simply reflect the different methodologies. It is well known that recognition tasks generally show greater retention for longer periods of time than do recall tasks.

\section{REFERENCES}

Bartram, D. J. Post-iconic visual storage: Chunking in the reproduction of briefly displayed visual patterns. Cognitive Psychology, 1978, 10, 324-355.

Cermak, G. W. Short-term recognition memory for complex freeform figures. Psychonomic Science, 1971, 25, 209-211.

Phillips, W. A. On the distinction between sensory storage and short-term visual memory. Perception \& Psychophysics, 1974, 16, 283-290.

Sperling, G. The information available in brief visual presentations. Psychological Monographs: General and Applied, 1960, 74, 1-29.

Wilton, R. N., \& File, P. E. Knowledge of spatial relations: A preliminary investigation. Quarterly Journal of Experimental Psychology, 1975, 27, 251-258.

(Received for publication September 25, 1980.) 